\title{
Assessing the predictive value of primary evaluation with the Immediate Post-Concussion Assessment and Cognitive Test following head injury
}

\author{
*Theodore Hannah, BA, ${ }^{1}$ Nickolas Dreher, BA, ${ }^{1}$ Adam Y. Li, BS, ${ }^{1}$ Dhruv S. Shankar, BS, ${ }^{1}$ \\ Ryan Adams, BS, ${ }^{1}$ Alex Gometz, DPT, CIC, ${ }^{2}$ Mark R. Lovell, PhD, ${ }^{3}$ and Tanvir F. Choudhri, MD ${ }^{1}$ \\ 1Department of Neurosurgery, Icahn School of Medicine at Mount Sinai, New York; ${ }^{2}$ Physical Medicine and Rehabilitation, \\ Concussion Management of New York, New York; and ${ }^{3}$ Department of Neurological Surgery, University of Pittsburgh Medical \\ Center, Pittsburgh, Pennsylvania
}

\begin{abstract}
OBJECTIVE Concussions are a major public health concern, especially for high school and college student athletes. However, there are few prognostic metrics that can accurately quantify concussion severity in order to anticipate recovery time and symptom regression. The Immediate Post-Concussion Assessment and Cognitive Test (ImPACT) is a widely used neurocognitive assessment that can diagnose and track recovery from concussions. This study assesses whether initial ImPACT scores, collected within 48 hours of the injury, can predict persistence of concussion at follow-up.

METHODS Results from 6912 ImPACT tests were compiled in 2161 unique student athletes, ages 12-22 years. The authors defined a novel metric, the Severity Index (SI), which is a summation of the number of standard deviations from baseline at the $80 \% \mathrm{Cl}$ for each of the 5 composite scores reported by ImPACT. Patients were binned into groups based on SI (0-3.99, 4-7.99, 8-11.99, 12+) and the relationships between SI groups, composite scores, symptom profiles, and recovery time were characterized using 1-way and 2-way ANOVAs and Kaplan-Meier plots. A logistic regression assessed the value of $\mathrm{SI}$ for predicting concussion at follow-up.

RESULTS Patients with a higher SI at diagnosis were more likely to still be concussed at their first follow-up $\left(\mathrm{F}_{3,2300}=\right.$ 93.06; $p<0.0001$ ). Groups with a higher SI also displayed consistently slower recovery over a 42-day period and were more likely to report symptoms in all 4 symptom clusters (Migraine, Cognition, Sleep, and Neuropsychiatric). When controlling for sex, age, number of previous concussions, days between assessments, and location, SI significantly increased the odds of being concussed at follow-up (OR 1.122, 95\% Cl 1.088-1.142; $p<0.001)$. This model showed good discrimination with an area under the curve of 0.74 .
\end{abstract}

CONCLUSIONS SI is a useful prognostic tool for assessing head injury severity. Concussions with higher initial SI tend to last longer and have broader symptomatic profiles. These findings can help patients and providers estimate recovery based on similar ImPACT score profiles.

https://thejns.org/doi/abs/10.3171/2020.2.PEDS19709

KEYWORDS concussion; head injury; trauma; neuropsychological testing; ImPACT; Immediate Post-Concussion Assessment and Cognitive Test; recovery; return to play; prognosis

$\mathrm{H}$ EAD injuries and concussions are a major public health concern, especially for high school and college student athletes., ${ }^{1,2}$ The Centers for Disease Control and Prevention found that in 2017, 15.1\% of students (approximately 2.5 million) reported suffering at least 1 concussion related to sports or physical activity in the past year. Furthermore, $40 \%$ of those students reported having 2 or more concussions in that period. ${ }^{3}$ Proper diagnosis and management are essential to reduce morbidity and reinjury rates. Terwilliger et al. showed that adolescent athletes who sustained an additional impact shortly after their initial concussion had significantly longer recovery

ABBREVIATIONS AUC = area under the curve; ImPACT = Immediate Post-Concussion Assessment and Cognitive Test; PCSS = Post-Concussion Symptom Scale; PI1 = first postinjury test; $\mathrm{PI} 2=$ second postinjury test; $\mathrm{S}_{\text {diff }}=$ standard error of difference at the $80 \% \mathrm{Cl}$; SI = Severity Index; $\Delta$ Score $=$ changes in composite scores.

SUBMITTED November 27, 2019. ACCEPTED February 5, 2020.

INCLUDE WHEN CITING Published online May 8, 2020; DOI: 10.3171/2020.2.PEDS19709.

${ }^{*}$ T.H. and N.D. contributed equally to this work. 
TABLE 1. Demographic data in 2304 patients with head injuries

\begin{tabular}{lccc}
\hline \multicolumn{1}{c}{ Variable } & Male & Female & Total \\
\hline No. of patients & 1528 & 776 & 2304 \\
\hline Age in yrs* & $15.87 \pm 1.61$ & $15.91 \pm 1.53$ & $15.88 \pm 1.58$ \\
\hline No. of concussions & 835 & 475 & 1310 \\
\hline
\end{tabular}

${ }^{*}$ Values are expressed as the mean $\pm \mathrm{SD}$.

time. ${ }^{4}$ Thus, health providers are constantly looking for new tools that will help them detect concussions early and track the individual's recovery accurately while navigating the complex landscape of physical and academic demands that predominate in the lives of student and professional athletes.

There has been extensive research regarding proper management of concussions, and computerized neurocognitive testing has emerged as a widely used and validated tool across high school, college, and professional sports teams. The most prevalent of these tests, the Immediate Post-Concussion Assessment and Cognitive Test (ImPACT), has demonstrated $81.9 \%$ sensitivity and $89.4 \%$ specificity in concussion diagnosis. ${ }^{5-7}$ It is also routinely used to track recovery as patients' symptoms gradually improve and their cognitive test scores return to baseline. However, few researchers have investigated the prognostic value of early ImPACT tests to determine if recoveries adhere to predictable patterns. Several studies have investigated the prognostic value of symptom profiles and have determined that patients who present with more severe symptoms take longer to recover. ${ }^{8-11}$ In 2011, Lau et al. attempted to improve on these predictions by incorporating postinjury ImPACT scores. ${ }^{6}$ These scores offer several standardized variables by which to assess concussion severity and thus estimate recovery time. These authors found that neurocognitive testing in conjunction with symptom clusters resulted in improved positive and negative predictive value for anticipating protracted recovery (longer than 14 days). ${ }^{6}$ However, studies that have attempted to assess the prognostic value have limited themselves to binary markers of recovery (i.e., long or short). Providers require more granularity if they want to use these predictions to help patients who may need to plan team rosters, request academic exemptions, obtain sick leave, or navigate various other practical concerns.

The primary aim of our analysis was to evaluate the prognostic value of the first ImPACT postinjury test on a variety of outcomes to help providers estimate recovery based on early markers of concussion severity. This analysis utilized one of the largest samples of ImPACT test results ever repurposed for research, allowing for more granular prognostic estimates. In order to more accurately capture head injury severity, we developed a novel, composite variable that measures the magnitude of significant, adverse changes on postinjury assessments from baseline: the Severity Index (SI). In this study, we leveraged SI to 1) characterize patient recovery across symptom clusters and ImPACT composite scores at multiple time points and 2) assess the likelihood of a patient having a concussion at their second postinjury test (PI2) based on SI at their first
TABLE 2. Distribution of sports in patients with concussions

\begin{tabular}{lcc}
\hline \multicolumn{1}{c}{ Sport } & No. of Patients & No. of Concussions \\
\hline Football & 683 & 357 \\
\hline Soccer & 200 & 112 \\
\hline Basketball & 104 & 56 \\
\hline Lacrosse & 104 & 56 \\
\hline Wrestling & 72 & 48 \\
\hline Volleyball & 64 & 39 \\
\hline Cheerleading & 54 & 33 \\
\hline Other & 123 & 76 \\
\hline Blank & 900 & 533 \\
\hline
\end{tabular}

postinjury test (PI1). By developing an intuitive metric to gauge concussion severity and testing its prognostic value, we hope to help athletes understand how their individual recovery is likely to manifest based on trends from similar injuries.

\section{Methods \\ Data Collection}

Data from 25,815 ImPACT tests conducted between 2009 and 2019 were provided through a research agreement with ImPACT Applications, Inc., which compiled de-identified data from 2 large, multicenter institutions. The subjects ranged in age from 12 to 22 years and resided in Florida $(19,833)$ or Colorado (5982). There were a total of 7445 tests conducted immediately after a head injury by a trained ImPACT administrator, usually a physician or athletic trainer. Of those 7445 PI1 tests, 4533 of the patients had a corresponding baseline test that could be used to make a concussion diagnosis and, of these subjects, 2304 had at least 1 follow-up test (PI2) after PI1 that could be used to assess recovery. Our analyses in this paper were restricted to those 2304 head injuries, of which 1310 resulted in a concussion diagnosis at PI1. In this subset of data there were 2161 unique patients, and the number of distinct head injuries suffered by each patient ranged from 1 to 5. Demographic data and the distribution of sports are summarized in Tables 1 and 2, respectively.

\section{Concussion}

The ImPACT test conducts a Post-Concussion Symptom Scale (PCSS) survey before testing the neurocognitive function of the patient using a variety of tests including word memory; design memory; X's and O's; and three-letter working memory, symbol-matching, and color-matching tasks over a 20 -minute session. ${ }^{5}$ Using a variety of metrics, the ImPACT test evaluates changes from baseline in 5 composite scores to provide a surrogate marker for concussion. Specifically, changes from baseline that exceeded the previously defined standard error of difference at the $80 \% \mathrm{CI}\left(\mathrm{S}_{\mathrm{diff}}\right)$ for healthy control subjects were considered significant. ${ }^{5} \mathrm{~A}$ patient is deemed to have suffered a concussion when a head injury results in changes from baseline that meet or exceed $S_{\text {diff }}$ in at least 2 of the 5 metrics. The composite scores are Verbal Memory 
TABLE 3. Distribution of patients in SI and recovery time groups

\begin{tabular}{lccccc}
\hline \multicolumn{1}{c}{ SI } & Total No. of Patients & 1-7 Days, No. (\%) & 8-14 Days, No. (\%) & 15-30 Days, No. (\%) & >30 Days, No. of Patients Excluded (\%) \\
\hline $0-3.99$ & 1240 & $840(67.8)$ & $231(18.6)$ & $97(7.8)$ & $72(5.8)$ \\
\hline $4-7.99$ & 523 & $299(57.1)$ & $142(27.2)$ & $65(12.4)$ & $17(3.3)$ \\
\hline $8-11.99$ & 269 & $142(52.8)$ & $77(28.6)$ & $36(13.4)$ & $14(5.2)$ \\
\hline $12+$ & 272 & $125(46.0)$ & $99(36.4)$ & $34(12.5)$ & $14(5.1)$ \\
\hline Total & 2304 & $1406(61.0)$ & $549(23.8)$ & $232(10.1)$ & $117(5.1)$ \\
\hline
\end{tabular}

$\left(\mathrm{S}_{\mathrm{diff}}=8.75\right)$, Visual Memory $\left(\mathrm{S}_{\mathrm{diff}}=13.55\right)$, Reaction Time $\left(\mathrm{S}_{\mathrm{diff}}=0.06\right)$, Processing Speed $\left(\mathrm{S}_{\mathrm{diff}}=4.98\right)$, and PCSS $\left(\mathrm{S}_{\mathrm{diff}}=9.18\right) .{ }^{5}$ The composition of these scores has been previously described. ${ }^{5}$

\section{Severity Index}

Changes in composite scores from baseline to PI1 ( $\Delta$ Score) were calculated as the absolute difference between the 2 scores (eq. 1). If $\Delta$ Score did not exceed $S_{\text {diff }}$, it was assumed that there was no change from baseline (eq. 2). To estimate the severity of each head injury, we summed the number of $S_{\text {diff }}$ values above baseline for each composite score to create an SI (eq. 3).

$$
\begin{aligned}
& \Delta \text { Score }=\mid \text { PI1 Score }- \text { Baseline Score } \mid \quad[\text { eq. 1] } \\
& f(\Delta \text { Score })=\left\{\begin{array}{ll}
0, & \Delta \text { Score }<\text { Sdiff } \\
\Delta \text { Score }, & \Delta \text { Score } \geq S \text { diff }
\end{array} \quad\right. \text { [eq. 2] } \\
& \text { Severity Index }=\sum_{i=1}^{5} \frac{f(\Delta \text { Score })_{i}}{\text { Sdiff }_{i}}
\end{aligned}
$$

Patients were binned into 4 groups based on SI (0-3.99 [n = 1240], 4-7.99 [ $\mathrm{n}=523], 8-11.99$ [n = 269], and 12+ [n = 272]) and used to examine differences in recovery based on SI at PI1. The SI is a continuous variable-the lower bound of each group is inclusive, and the upper bound is exclusive. An SI calculator is provided in Table S4 for health providers seeking to use this metric in their practice.

\section{Recovery Time Analyses}

To evaluate differences in recovery over time, the SI bins were subdivided into 3 categories based on how long after PI1 the patient took PI2 (1-7 days, 8-14 days, or 15-30 days). Patients with PI2s that did not occur within these time frames were excluded. The number of patients in each group is summarized in Table 3.

\section{PCSS Score Clusters}

The PCSS score clusters were calculated as previously described. ${ }^{6,12}$ Briefly, the PCSS score is the sum of 22 standardized symptoms rated on a scale from 0 to 7 by the patient and is used as 1 of the 5 ImPACT composite scores. However, previous researchers have broken the PCSS score into 4 clusters: Migraine, Cognitive, Sleep, and Neuropsy- chiatric. The Migraine cluster includes headaches, visual problems, dizziness, noise sensitivity, light sensitivity, balance problems, numbness/tingling, nausea, and vomiting. The Cognitive cluster includes fatigue, fogginess, drowsiness, cognitive slowing, difficulty concentrating, and difficulty remembering. The Sleep cluster includes difficulty sleeping, sleeping more than usual, and sleeping less than usual. The Neuropsychiatric cluster includes feeling more emotional, experiencing sadness, experiencing nervousness, and experiencing irritability. The range of possible scores is 0-63 for the Migraine cluster, 0-42 for the Cognitive cluster, 0-21 for the Sleep cluster, and 0-28 for the Neuropsychiatric cluster.

\section{Kaplan-Meier Plots}

Kaplan-Meier plots were used to examine concussion recovery patterns in aggregate. The event in these survival analyses was defined as recovery from a concussion. For these analyses, all follow-up tests conducted within 42 days of PI1 for each patient were used. All patients had between 1 and 3 tests within that window. These analyses were restricted to the 1310 patients who had a concussion at PI1.

\section{Logistic Regression}

The logistic regression analysis was performed with Python. In order to include number of previous concussions as a control variable, our analysis was restricted to 1325 patients.

\section{Statistical Analyses}

All statistical analyses other than the logistic regression were performed with Prism 8.0 (GraphPad Software). One-way ANOVA was used to compare the means between SI groups. Two-way ANOVA was used to compare differences between severity scores and recovery time points. For both 1-way and 2-way ANOVA, post hoc Tukey tests were used to conduct univariate analyses and determine significant differences. Log-rank (Mantel-Cox) tests were used to evaluate significant differences between Kaplan-Meier curves. For all analyses, $\alpha=0.05$.

\section{Results \\ Demographics}

The data set used for these analyses contains baseline, PI1, and PI2 ImPACT assessments for 2304 head injuries. The average age of the patients at the time of their injury was $15.88 \pm 1.58$ years, and of the 2304 with head inju- 


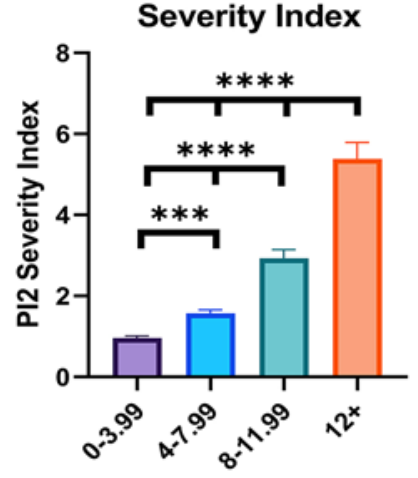

A PI1 Severity Index

Significant $\Delta$ Scores

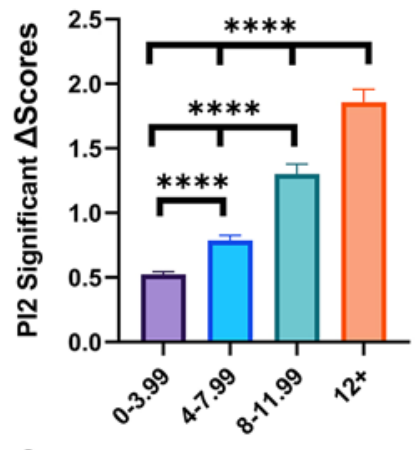

C PI1 Severity Index

FIG. 1. Associations between SI at PI1 and concussions at PI2. A: The mean SI at PI2 grouped by SI at PI1. B: Percentage of patients with a concussion at PI2. C: The mean number of the 5 composite scores used to diagnose concussion that deviated from baseline by at least $S_{\text {diff }}$. D: The mean number of days between the patients' PI1 and PI2 tests. Multipronged significance bars denote significant differences between the value under the prong on the far right and all values under the other prongs. Error bars indicate SEM. ${ }^{* *} p<0.01 ;{ }^{* * *} p<0.001 ;{ }^{* * * *} p<0.0001$. Figure is available in color online only.

ries, $1310(57 \%)$ were diagnosed with a concussion during their PI1 screening. In our cohort, 1528 of the patients were male with an average age of $15.87 \pm 1.61$ years and 776 of the patients were female with an average age of $15.91 \pm 1.53$ years. A concussion was diagnosed at PI 1 in $835(55 \%)$ of the males and $475(61 \%)$ of the females. The most common sport played by patients in the data set was football $(n=683)$, followed by soccer $(n=200)$, basketball $(n=104)$, and lacrosse $(n=104)$. No sport was listed for 900 patients (Table 2).

\section{SI at PI1 Correlates With Percentage of Patients Who Remained Concussed at PI2}

To assess whether our metric for estimating concussion severity was consistent over time, we tested whether patients with higher PI1 severity scores had consistently higher PI2 severity scores, higher diagnosed concussion rates at PI2, and higher numbers of significant $\Delta$ Scores (Fig. 1). We found that patients with a higher SI at PI1 did continue to experience a higher SI at PI2 $\left(\mathrm{F}_{3,2300}=171.0\right.$; $\mathrm{p}<0.0001)$ and that differences between all groups were significant (Fig. 1A). The number of patients who still had
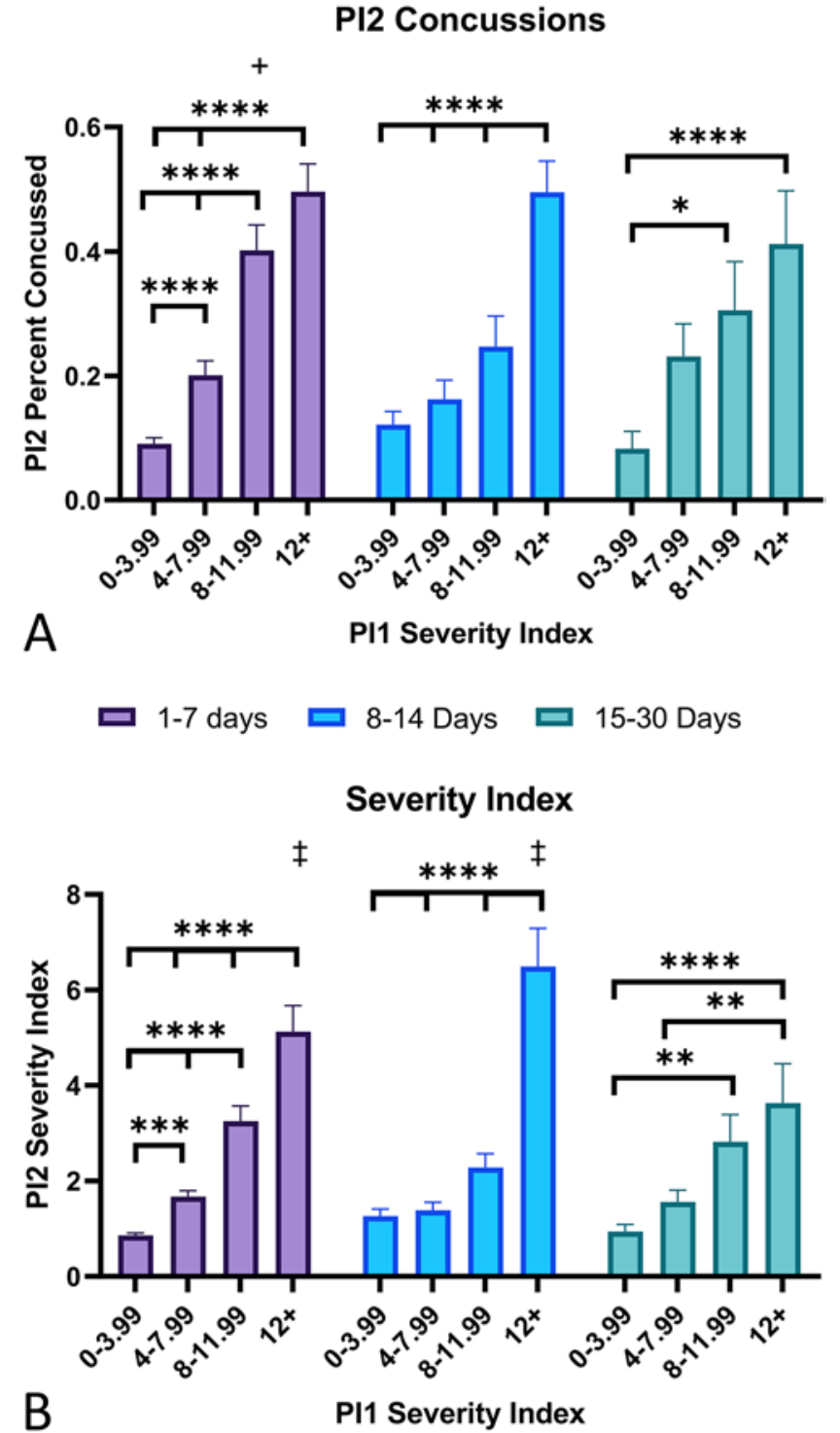

FIG. 2. Associations between SI at PI1 and concussions at PI2 subdivided by recovery time. A: Percentage of patients with a concussion at PI2 for 3 different recovery time intervals. B: The mean SI at PI2 in patients with different SIs at PI1 at 3 different recovery time intervals. Multipronged significance bars denote significant differences between the value under the prong on the far right and all values under the other prongs. Error bars indicate SEM. The + symbol indicates significant difference from the same SI at the 8- to 14-day time point. The $\ddagger$ symbol indicates significant difference from the same SI at both other time points. ${ }^{*} p<0.05 ;{ }^{* *} p<0.01 ;{ }^{* * *} p<0.001 ;{ }^{* * * *} p<0.0001$. Figure is available in color online only.

a diagnosable concussion at PI2 was also higher $\left(\mathrm{F}_{3,2300}=\right.$ 93.06; $\mathrm{p}<0.0001)$ in patients who had a higher SI at PI1 (Fig. 2B). Additionally, the number of significant $\Delta$ Scores at PI2 was higher $\left(\mathrm{F}_{3,2300}=154.1 ; \mathrm{p}<0.0001\right)$ in patients who had higher PI1 severity scores (Fig. 1C). To ensure that these analyses were not biased by the number of days between tests, we calculated how many days elapsed between PI1 and PI2 for each patient. A 1-way ANOVA revealed a significant difference $\left(\mathrm{F}_{4,2299}=4.615 ; \mathrm{p}=0.0032\right)$ between groups; however, a post hoc Tukey test showed 


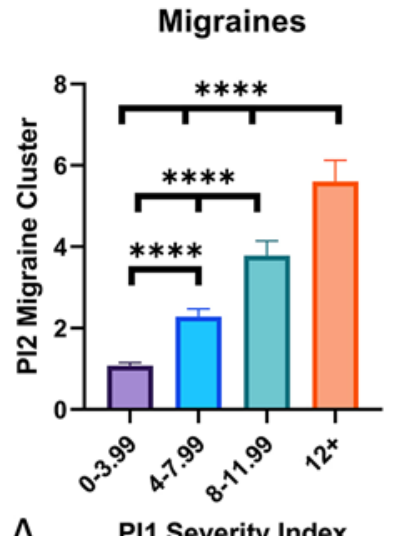

A

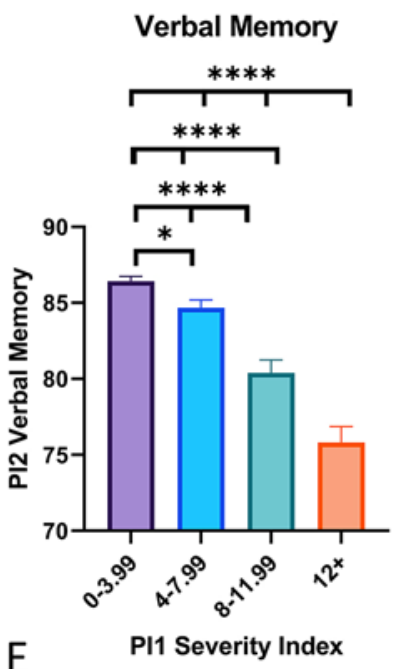

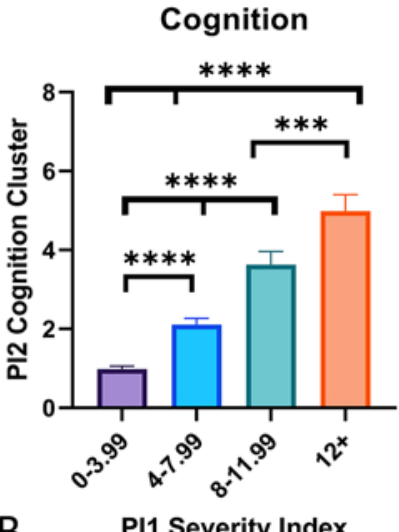

B

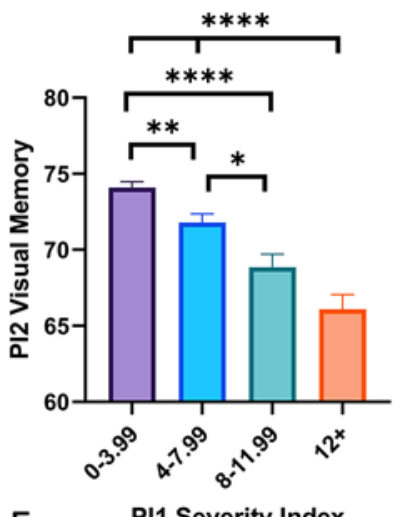

$\mathrm{F}$

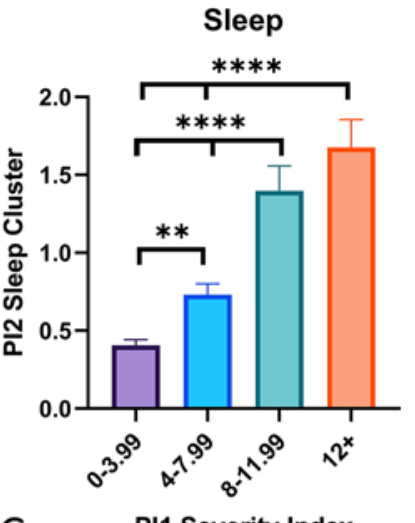

C

Processing Speed

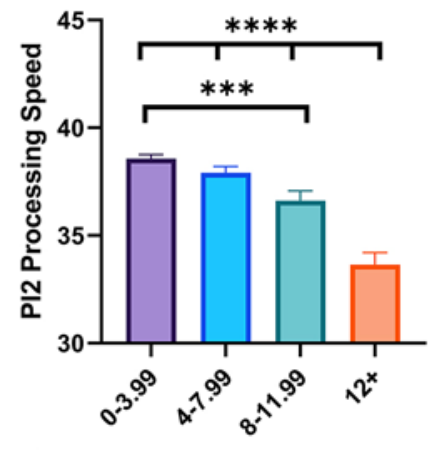

G

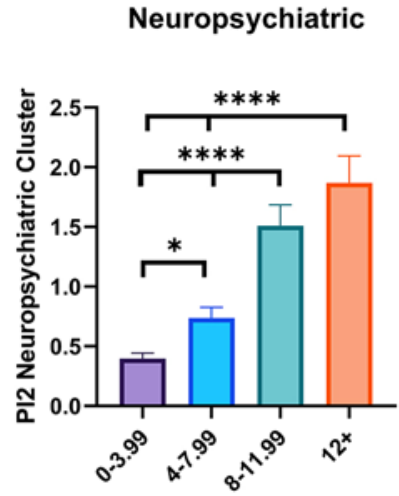

D

PI1 Severity Index

FIG. 3. Symptom cluster and ImPACT composite scores at PI2. The mean scores from PI2 tests for patients suffering a head injury based on initial SI of the injury. A: Migraine cluster scores. B: Cognition cluster scores. C: Sleep cluster scores. D: Neuropsychiatric cluster scores. E: Verbal Memory composite scores. F: Visual Memory composite scores. G: Processing Speed composite scores. H: Reaction Time composite scores. Multipronged significance bars denote significant differences between the value under the prong on the far right and all values under the other prongs. Error bars indicate SEM. ${ }^{*} p<0.05 ;{ }^{* *} p<0.01 ;{ }^{* * *} p<0.001$; ${ }^{* * * *} p<0.0001$. Figure is available in color online only.

that the only difference was that patients in the $0-4$ group took significantly fewer days between PI1 and PI2 tests than did those in the 12+ group (Fig. 1D). This bias acts in the opposite direction of our results, so it does not impact the validity of the results.

However, it is still possible that the trends seen in Figs. 1 and 3 are driven by patients who took PI2 so close to PI1 that there was effectively no recovery time, resulting in PI2 being indistinguishable from PI1. To address the possibility of such a circular analysis, we reanalyzed the data based on recovery time. Specifically, we looked at aggregate PI 2 scores during 3 different time points: PI 2 tests taken 1-7 days after PI1, 8-14 days after PI1, and 15-30 days after PI1 (Fig. 2). The number of patients for each SI group who took PI2 at each interval are listed in Table 3. Patients with a lower SI were more likely to take their PI2 test within 7 days of PI1 than were patients with a higher SI. The percentage of patients who took PI2 8-14 days after PIl increased with initial SI. A similar percentage of patients in all groups, except 0-4, waited 15-30 days to take PI2.

We found that head injuries that initially presented with a higher SI resulted in increased percentages of concussion diagnosis at each time point. The percentage of patients diagnosed with a concussion with an initial SI of 0-3.99 or 4-7.99 was stable (approximately $8 \%$ and 19\%, respectively) across the first 14 days (Fig. 2A). In contrast, the percentage of patients with an initial SI of 8-11.99 who remained concussed 8-14 days after PI1 (24\%) was significantly lower $(\mathrm{p}=0.0092)$ than the percentage who were still concussed 1-7 days after PI1 (40\%). Patients with an initial SI of 12 or greater also had similar rates ( $p$ $>0.9999$ ) of concussion lasting at least 8-14 days (50\%) or 1-7 days (50\%). The percent concussed for this group decreased to $41 \%$ for patients who waited 15-30 days to take PI2, although this decrease was not statistically significant $(\mathrm{p}=0.9935)$. A 2-way ANOVA revealed that the main effect of SI $\left(\mathrm{F}_{3,2175}=52.98 ; \mathrm{p}<0.0001\right)$ and the in- 
teraction between SI and time $\left(\mathrm{F}_{2,2175}=2.166 ; \mathrm{p}=0.0459\right)$ were significant. The main effect of time $\left(\mathrm{F}_{2,2175}=2.142 ; \mathrm{p}\right.$ $=0.0459$ ) was not significant.

The SI at PI2 was also positively correlated with the SI at PI1 across all time points. For patients with an initial SI lower than 12, the mean SI recorded at PI2 was similar at every interval (Fig. 2B). However, the SI at PI2 for patients in the 12+ group was markedly higher at 8-14 days (mean 6.49) than it was at 1-7 days (mean 5.13) or at 15-30 days (mean 3.63). A 2-way ANOVA revealed a significant interaction between SI and time $\left(\mathrm{F}_{6.2175}=5.092 ; \mathrm{p}<0.0001\right)$ and a significant main effect of $\mathrm{SI}\left(\mathrm{F}_{3,2175}=92.82 ; \mathrm{p}<0.0001\right)$. The main effect of time $\left(\mathrm{F}_{2,2175}=2.922 ; \mathrm{p}=0.0541\right)$ was not significant.

\section{SI Is Associated With Higher Symptom Cluster Scores at PI2}

Certain symptom clusters and ImPACT composite scores have shown predictive value for the length of time to recovery from concussion. To examine the trends in the symptom clusters between different levels of severity, we calculated the average symptom cluster scores at PI 2 for the Migraine, Cognitive, Sleep, and Neuropsychiatric clusters (Fig. 3A-D). Additionally, we looked at the relationship between SI at PI1 and the 4 other ImPACT composite scores at PI2: Verbal Memory, Visual Memory, Processing Speed, and Reaction Time (Fig. 3E-H). The results of the 1 -way ANOVAs are listed in Table S1. Significant differences between scores for 0-4 and 4-8 were observed in all metrics except Processing Speed. Conversely, for the 2 highest SI groups, no significant differences were found. The SI 4-7.99 group had significantly lower reported symptoms and functional deficits for each metric except Processing Speed and Reaction Time.

To address the possibility that the trends displayed in Fig. 3 were a result of patients taking PI2 so close to PI1 that they experienced no recovery time, we looked at trends at the same 3 time points as in Fig. 2. The results demonstrate that the trends seen in Fig. 3 hold in all metrics for up to 14 days after PI1 and in most metrics for up to 30 days (Fig. S3). Additionally, it appears that the spike in SI at PI2 taken 8-14 days after PI1 for the 12+ group shown in Fig. 2B is driven by significantly higher impairment in Verbal Memory and Reaction Time tasks as well as increased symptom scores in all clusters. There is more variability at the 15 - to 30 -day time point, probably driven in part by low sample sizes. However, the 12+ SI group consistently appears to have worse symptoms and composite scores than the $0-3.99,4-7.99$, and $8-11.99$ groups, although there is often no statistical significance. Using 2-way ANOVA, all metrics were found to have significant main effects on SI. Cognitive and Verbal Memory had significant main effects on time between PI1 and PI2. There were no significant interaction effects. ANOVA results of these analyses are summarized in Table S2.

\section{SI Predicts Length of Time to Recover From Concussion}

To assess aggregate recovery rates over time, we used Kaplan-Meier plots to display how long patients took to recover from their concussions. At PI1, the percentages of patients with a diagnosed concussion were $24 \%, 91 \%$,
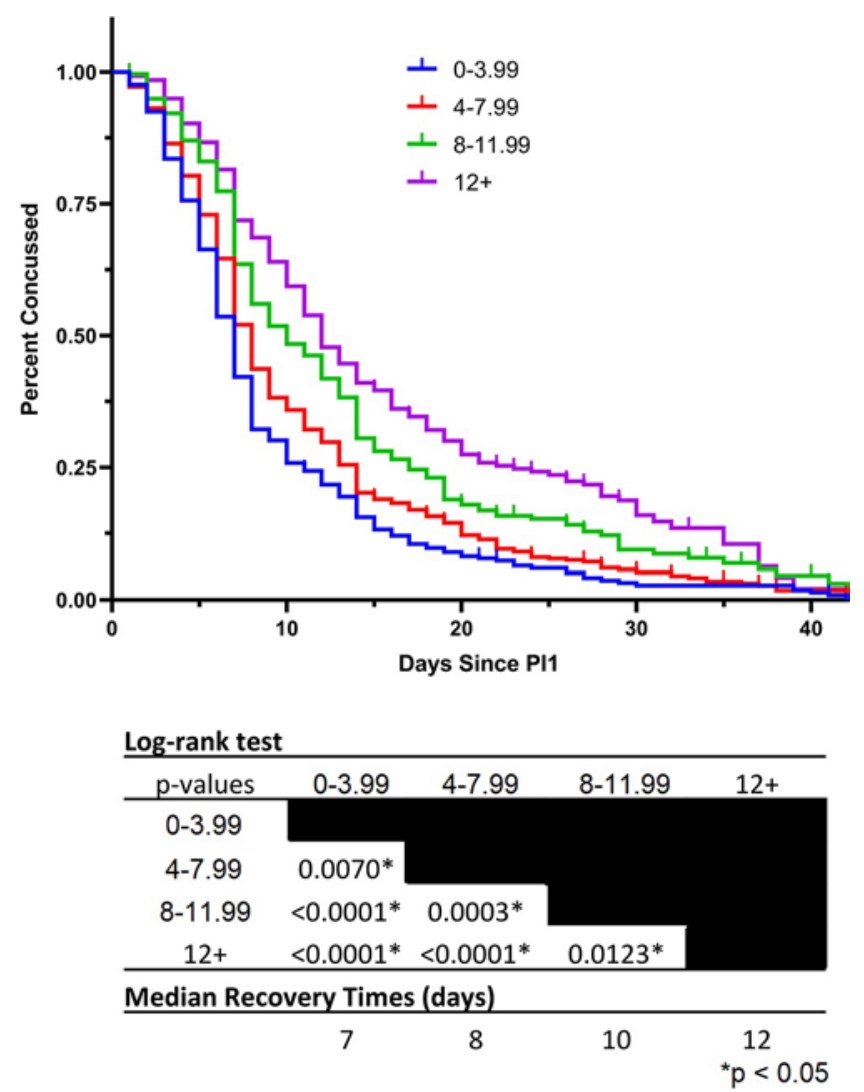

FIG. 4. Concussion recovery for patients with a diagnosed concussion at PI1. Upper: Kaplan-Meier plot for each SI group showing the percentage of patients who are still concussed at their follow-up tests from 1 to 42 days after taking their PI1 test. Lower: Log-rank test $p$ values for differences between curves and median recovery time in days are listed below the plots. ${ }^{*} p<0.05$. Figure is available in color online only.

$98 \%$, and $100 \%$ for SI groups $0-3.99,4-7.99,8-11.99$, and $12+$, respectively. For each of these patients diagnosed with a concussion at PI1, we followed their recovery for up to 42 days (Fig. 4). We used log-rank tests to determine whether the curves differed from each other and found that the curves were all significantly different. The median concussion recovery times were 7 days, 8 days, 10 days, and 12 days.

Finally, to assess the value of SI as a predictive factor for length of time to concussion recovery, we performed a logistic regression to predict which patients would be concussed at PI 2 by using SI at PI1 while controlling for sex, age, number of previous concussions, days between PI1 and PI2, and location (Colorado or Florida). We found that SI (OR 1.122, 95\% CI 1.088-1.142) significantly increased the odds of being concussed at PI2, whereas being older (OR 0.875, 95\% CI 0.857-0.892) decreased the odds of being concussed at PI2 (Table 4). The area under the curve (AUC) for the model was 0.74 .

\section{Discussion}

We have introduced a novel metric, the SI, and used it to assess the prognostic value of PI1 ImPACT tests in a multisport population of 2304 head injuries. Patients 
TABLE 4. Results of logistic regression predicting concussion at $\mathrm{PI} 2$ by using SI at PI1

\begin{tabular}{lccr}
\hline Logistic Regression & OR & $95 \% \mathrm{Cl}$ & $\mathrm{p}$ Value \\
\hline Sex & 0.851 & $0.602-1.206$ & 0.365 \\
\hline Age & 0.875 & $0.857-0.892$ & $<0.001$ \\
\hline Concussion history & 1.055 & $0.875-1.27$ & 0.573 \\
\hline SI & 1.122 & $1.088-1.142$ & $<0.001$ \\
\hline Days btwn PI1 \& PI2 & 0.998 & $0.993-1.003$ & 0.374 \\
\hline Location & 1.029 & $0.691-1.531$ & 0.887 \\
\hline
\end{tabular}

Boldface type indicates statistical significance.

with a higher SI were more likely to still be concussed at the time of their PI2, and higher SI groups display slower recovery consistently over a 42-day period. Patients with a higher SI were also more likely to report symptoms in all 4 symptom clusters (Migraine, Cognition, Sleep, and Neuropsychiatric) and worse composite scores for each of the ImPACT domains (Verbal Memory, Visual Memory, Processing Speed, and Reaction Time). SI was also found to be a good predictor of which patients would still have a concussion at PI2. Thus, a higher SI was highly correlated with worse symptom profiles across time and with longerlasting concussions.

Previous studies have found that individual, severe concussion symptoms noted at the time of injury, such as amnesia or loss of consciousness, do not correlate closely with recovery time. ${ }^{13,14}$ However, various studies have shown that increased aggregate symptom severity immediately following concussion is associated with longer recovery times. $^{8-11}$ For example, Miller et al. found that children were at higher risk for postconcussive symptoms lasting more than 28 days if they presented with higher scores on their initial symptom assessment (adjusted OR 5.58, 95\% CI 2.61-11.93). ${ }^{10}$ Heyer et al. studied 1953 youths with concussions and found that symptom severity on the day of clinic evaluation predicted symptom duration. ${ }^{9}$ As a result, symptom severity scores have been a primary prognostic indicator for concussions. ${ }^{13-15}$ The present study corroborates these findings-aggregate symptom severity scores were included in the calculation of SI, and initial SI was found to be a beneficial tool for assessing recovery prognosis. Moreover, this study showed that a higher SI is associated with longer-lasting symptoms in all 4 symptom clusters across time-a result not previously elucidated in ImPACT predictive modeling literature. .,12,16,17 $^{-17}$

Waiting for regression of concussion symptoms is currently the preferred method for determining when to begin return-to-play protocols. ${ }^{14,18,19}$ One rationale for this is that it is thought that symptoms last longer than the cognitive impairments associated with concussion. ${ }^{14,18}$ However, neurocognitive deficits, considered to be objective measures of concussion, have proven to be more sensitive than symptoms alone in the diagnosis of concussion. ${ }^{5,7,14,20}$ Furthermore, neurocognitive composite scores may provide additional value in predicting trends in concussion recovery. ${ }^{12,21,22}$ For example, in 2011 Lau et al. used ImPACT neurocognitive composite scores to predict recovery taking longer than 14 days (discriminant function analysis: $\left.\mathrm{F}_{4,99}=3.946 ; \mathrm{p}<0.005\right){ }^{6}$ In the present study, Kaplan-Meier analyses showed that patients with high SI were less likely to be recovered at all time points up to 42 days. Thus, this study demonstrates, at a more detailed level than previous research, that cognitive function scores from the ImPACT test can be used to estimate recovery time. Moreover, the inclusion of neurocognitive function data in our SI metric may be of particular importance for students making return-to-learn decisions such as missing school or postponing examinations.

Combining symptom and neurocognitive scores into one metric, SI, increased the discriminative capacity of models predicting length of time to recovery compared with symptoms or individual neurocognitive scores alone. The models for predicting protracted recovery in another study by Lau et al. (in 2012) had AUCs ranging from 0.45 to 0.66 for individual neurocognitive and symptom components, whereas our SI model achieved an AUC of 0.74.12 This difference in AUC is probably a direct result of the fact that SI merges multiple predictors into one variable, capturing differences in severity that are missed by singlefactor models used in previous studies. For example, in assessing the predictive value of Verbal Memory in other models, a concussion that results in Verbal Memory, Visual Memory, and Processing Speed scores all 4 SDs below baseline (SI 12) is considered equivalent to a concussion in which Verbal Memory scores deviate from baseline by 4 SDs, but Visual Memory only deviates by 1 SD and Processing Speed does not deviate at all (SI 5). Nevertheless, our analyses indicate that those 2 patients will have significantly different recovery experiences. Using a larger sample and a novel metric of concussion severity, we have shown that deviations from baseline in symptom and neurocognitive scores are more predictive of recovery time than previously understood. Additionally, this study is the first to stratify prognostic estimates across several severity levels, allowing for more personalized recovery predictions.

Although SI improves on previous models of concussion recovery prediction, these other models provide evidence that SI might be enhanced by applying different weights to variables in accordance with their predictive value. ${ }^{12,17}$ For example, in 2012 Lau et al. showed that the Migraine symptom cluster and Visual Memory composite score have higher predictive value (AUCs 0.66) than the Neuropsychiatric symptom cluster (AUC 0.53) and Verbal Memory composite score (AUC 0.45 ). In its current form, SI gives equal influence to each of the 5 composite scores, and each of the symptom score clusters is also equally weighted with respect to the others. Future studies should try to improve the predictive utility of SI by modulating the influence of the composite scores as deemed appropriate by rigorous statistical analyses. Additionally, this study only examined sports-related concussions in adolescent athletes. Going forward, the generalizability of these results to all types of pediatric concussion should be evaluated.

\section{Limitations of the Study}

Although we were able to capture patient recovery through follow-up testing at PI2, this does not give the ex- 
act duration of concussion because the patient could have recovered in the days prior to the test. Thus, our KaplanMeier analysis may overestimate recovery time. Furthermore, we do not know how long it took patients to recover if they still had a concussion at their last follow-up, because this is our primary endpoint. These factors limit our ability to predict concussion duration, particularly in the long term.

\section{Conclusions}

By defining the SI and validating its relationship with concussion duration, we have established a foundation for more specific, predictive modeling. Many providers may already assume that larger deviations from baseline indicate a more severe and long-lasting concussion, but research confirming this hypothesis is essential given the variability in concussion presentation. Until such prognostic tools are developed, athletes and providers can use these data to approximate recovery time by examining trends for patients with similar SIs. Any insight into recovery that can be derived in the acute phase after injury is invaluable for patients attempting to map out the weeks following their concussion.

\section{References}

1. Ianof JN, Freire FR, Calado VTG, et al. Sport-related concussions. Dement Neuropsychol. 2014;8(1):14-19.

2. Langlois JA, Rutland-Brown W, Wald MM. The epidemiology and impact of traumatic brain injury: a brief overview. $J$ Head Trauma Rehabil. 2006;21(5):375-378.

3. DePadilla L, Miller GF, Jones SE, et al. Self-reported concussions from playing a sport or being physically active among high school students-United States, 2017. MMWR Morb Mortal Wkly Rep. 2018;67(24):682-685.

4. Terwilliger VK, Pratson L, Vaughan CG, Gioia GA. Additional post-concussion impact exposure may affect recovery in adolescent athletes. $J$ Neurotrauma. 2016;33(8):761-765.

5. Iverson GL, Lovell MR, Collins MW. Interpreting change on ImPACT following sport concussion. Clin Neuropsychol. 2003;17(4):460-467.

6. Lau BC, Collins MW, Lovell MR. Sensitivity and specificity of subacute computerized neurocognitive testing and symptom evaluation in predicting outcomes after sports-related concussion. Am J Sports Med. 2011;39(6):1209-1216.

7. Schatz P, Pardini JE, Lovell MR, et al. Sensitivity and specificity of the ImPACT Test Battery for concussion in athletes. Arch Clin Neuropsychol. 2006;21(1):91-99.

8. Fehr SD, Nelson LD, Scharer KR, et al. Risk factors for prolonged symptoms of mild traumatic brain injury: a pediatric sports concussion clinic cohort. Clin J Sport Med. 2019;29(1):11-17.

9. Heyer GL, Schaffer CE, Rose SC, et al. Specific factors influence postconcussion symptom duration among youth referred to a sports concussion clinic. J Pediatr. 2016;174:33-38.e2.

10. Miller JH, Gill C, Kuhn EN, et al. Predictors of delayed recovery following pediatric sports-related concussion: a casecontrol study. J Neurosurg Pediatr. 2016;17(4):491-496.

11. Thomas DJ, Coxe K, Li H, et al. Length of recovery from sports-related concussions in pediatric patients treated at concussion clinics. Clin J Sport Med. 2018;28(1):56-63.

12. Lau BC, Collins MW, Lovell MR. Cutoff scores in neurocognitive testing and symptom clusters that predict protracted recovery from concussions in high school athletes. Neurosurgery. 2012;70(2):371-379.
13. Meehan WP III, Mannix RC, Stracciolini A, et al. Symptom severity predicts prolonged recovery after sportrelated concussion, but age and amnesia do not. J Pediatr. 2013;163(3):721-725.

14. Teel EF, Marshall SW, Shankar V, et al. Predicting recovery patterns after sport-related concussion. J Athl Train. 2017;52(3):288-298.

15. Begasse de Dhaem O, Barr WB, Balcer LJ, et al. Post-traumatic headache: the use of the Sport Concussion Assessment Tool (SCAT-3) as a predictor of post-concussion recovery. $J$ Headache Pain. 2017;18(1):60.

16. Hang B, Babcock L, Hornung R, et al. Can computerized neuropsychological testing in the emergency department predict recovery for young athletes with concussions? Pediatr Emerg Care. 2015;31(10):688-693.

17. Resch JE, Brown CN, Macciocchi SN, et al. A preliminary formula to predict timing of symptom resolution for collegiate athletes diagnosed with sport concussion. J Athl Train. 2015;50(12):1292-1298.

18. McCrory P, Meeuwisse WH, Aubry M, et al. Consensus statement on concussion in sport: the 4th International Conference on Concussion in Sport held in Zurich, November 2012. Br J Sports Med. 2013;47(5):250-258.

19. Wolf C, Fast K. "Put me back in, coach!" Concussion and return to play. Mo Med.2017;114(1):36-39.

20. Dessy AM, Yuk FJ, Maniya AY, et al. Review of assessment scales for diagnosing and monitoring sports-related concussion. Cureus. 2017;9(12):e1922.

21. Erlanger D, Kaushik T, Cantu R, et al. Symptom-based assessment of the severity of a concussion. J Neurosurg. 2003;98(3):477-484.

22. Iverson GL, Gardner AJ, Terry DP, et al. Predictors of clinical recovery from concussion: a systematic review. $\mathrm{Br} \mathrm{J}$ Sports Med. 2017;51(12):941-948.

\section{Disclosures}

Dr. Lovell is the co-founder of ImPACT Applications, Inc.

\section{Author Contributions}

Conception and design: Dreher, Hannah, Li, Gometz, Lovell, Choudhri. Acquisition of data: Dreher, Hannah, Li, Adams, Gometz, Lovell, Choudhri. Analysis and interpretation of data: Dreher, Hannah. Drafting the article: Dreher, Hannah. Critically revising the article: all authors. Reviewed submitted version of manuscript: all authors. Approved the final version of the manuscript on behalf of all authors: Dreher. Statistical analysis: Dreher, Hannah. Administrative/technical/material support: all authors. Study supervision: Gometz, Lovell, Choudhri.

\section{Supplemental Information \\ Online-Only Content}

Supplemental material is available with the online version of the article.

Tables and Figures S1-S4. https://thejns.org/doi/suppl/10. 3171/2020.2.PEDS19709.

\section{Correspondence}

Nickolas Dreher: Icahn School of Medicine at Mount Sinai, New York, NY.nickolas.dreher@icahn.mssm.edu. 